\title{
Important considerations before Initiation of Ibrutinib Treatment
}

\author{
Nahla A M Hamed* \\ Professor of Hematology, Faculty of Medicine, Alexandria University, Egypt \\ Submission: July 15, 2019; Published: September 03, 2019 \\ ${ }^{*}$ Correspondence Author: Nahla A M Hamed, Professor of Hematology, Faculty of Medicine, Alexandria University, Egypt
}

\section{Abstract}

Ibrutinib is a Bruton's tyrosine kinase inhibitor which interferes with signaling through the B-cell receptor pathway. Ibrutinib is metabolized via cytochrome P450 enzyme 3A. Potential drug interactions need to be considered with its use. Ibrutinib is generally well tolerated. Diarrhea and skin rashes are relatively common, often transient, and can resolve with no specific management. Bleeding, atrial fibrillation, arthritis and arthralgia, fatigue, cytopenias, and infections are more serious but less common drug specific complications.

Abbreviations: AF: Atrial Fibrillation; CNS: Central Nervous System; PI3K: Phosphoinositide 3-Kinase; CYP3A: Cytochrome P450 enzyme 3A; AP: Antiplatelet; AC: Anticoagulant

\section{Introduction}

Ibrutinib is very well-tolerated in general. However, there are several adverse effects that warrant consideration prior to its initiation [1]. The most common side effects of ibrutinib are diarrhea, upper respiratory tract infection, bleeding, fatigue and cardiac side effects. These events are generally mild (grade I-II). However atrial fibrillation and bleeding are important and may be grade III or higher and require strict monitoring [2].

\section{Immunologic changes and the risk of Infection?}

Pneumonia, upper respiratory tract infections, sinusitis and urinary tract infections are the most commonly reported infections in pivotal CLL trials [3]. Infections were more frequent during the first 6 months and in relapsed/refractory CLL [4]. It is assumed that the infection rate will decrease with time and when ibrutinib is started earlier in CLL disease course therapy [3]. Patients with a $\geq 50 \%$ increase in serum IgA levels had a lower rate of infection [4]. Immune deficiency, such as hypogammaglobulinemia, contributes to the relatively high rates of infection and morbidity in CLL patients [3]. Ibrutinib treatment resulted in a partial reconstitution of the humoral immunity [5] and the normal B-cell subpopulations, rendering a lower rate of infection in CLL patients. Serum immunoglobulin G levels still decrease during treatment [5]. Effects of ibrutinib on dysregulation of the normal $\mathrm{T}$ and natural killer cell compartment still require an extensive investigation [4].

\section{Consider the following in patients on Ibrutinib}

\section{Prior to start Ibrutinib treatment}

i. Vaccinations (e.g. influenza and pneumonia) should be strongly encouraged [3].

ii. Testing for HBV serology before ibrutinib treatment initiation was suggested to be mandatory and preventive antiviral therapy for patients with a previous HBV infection should be considered [5].

\section{Once ibrutinib therapy has been Initiated}

a. Routine check at every clinic visit for fever, neutropenia and infections [3].

b. Infections prophylaxis was not recommended especially in the first line setting. Factors such as prior therapy, immune status, splenectomy and prior infections should be determined [3].

c. For low-grade infections, continue ibrutinib therapy [3]. Adjust the dose of clarithromycin, ketoconazole or voriconazole (moderate/strong CYP3A4 inhibitors) if used [3].

d. Coadministration of $140 \mathrm{mg}$ ibrutinib with voriconazole demonstrated an acceptable safety profile, and the adverse event profile was consistent with the ibrutinib safety profile at therapeutic doses [6]. 
e. Sulfamethoxazole and trimethoprim combination may be appropriate for patients previously received chemoimmunotherapy [3].

f. Vigorous treatment of emergent infections including the opportunistic infections, such as Pneumocystis (jirovecii) pneumonia. The use of growth factors should be considered [3].

\section{Bleeding Events}

CLL patients are at increased risk for major bleeding because of the disease itself, comorbid conditions, concomitant medications or clinically significant thrombocytopenia [7]. Bleeding events of any grade [8] have been reported in ibrutinibtreated patients [7] with a 2.93-fold increase compared to the control arms. Lower grade $(<3)$ bleeding (mainly ecchymosis and petechiae during the first 6 months) was reported in $28 \%$ of 50 patients not on simultaneous AP or AC therapy. None of the studied patients had major bleeding. Collagen-induced platelet aggregation was inhibited, whereas ADP induced platelet aggregation improved on ibrutinib therapy [8]. BTK and TEC are expressed in platelets and act downstream in glycoprotein (GP) VI signaling involved in collagen-mediated platelet aggregation. BTK is thought to play a more dominant role than TEC [7].

Platelet therapy should be initiated if the site of bleeding is non-CNS, even in non-thrombocytopenic patients. Platelet transfusions are not advised in the event of CNS bleeding. In emergency surgery, a platelet transfusion (to receive $50 \%$ fresh platelets) is appropriate, taking into consideration the timing of the last dose and the half-life of ibrutinib [3]. Precautions should be taken on concomitant use of anticoagulants and antiplatelet agents, along with adherence to perioperative ibrutinibwithholding guidelines [7].

\section{Cardiac Adverse Events}

The only significant cardiac adverse events (AEs) reported is grade 1-2 hypertension (observed in $18-29 \%$ of clinical trial patients) and atrial fibrillation (grade 3 or higher toxicity in $4 \%$ of patients on ibrutinib as compared to $1 \%$ on temsirolimus in phase III study) [9]. The increased risk of atrial fibrillation associated with ibrutinib may be related to on-target toxicity to the PI3K-Akt pathway in cardiomyocytes [8]. Most AF events typically occurred within the first 6 months after initiation of ibrutinib therapy and continued at a low rate over time [8]. Physicians should always consider the possibility of AF in patients who develop $\mathrm{CV} /$ respiratory symptoms while taking ibrutinib and have to perform an ECG [3]. The choice of anticoagulants in cases ibrutinib related AF is not clear enough [8].

\section{Lymphocytosis}

Ibrutinib is often associated with asymptomatic lymphocytosis $(70 \%)$ upon treatment initiation. Lymphocytosis is inherent to its mechanism of action, as ibrutinib disrupts integrin-mediated adhesion and homing of malignant B cells to the lymphoid microenvironment [8]. Lymphocytosis usually peaks after one month of therapy, and subsequently slowly declines. The median time to resolution of lymphocytosis on ibrutinib therapy is 19 weeks, but prolonged lymphocytosis up to 124 weeks has been seen in patients with ongoing treatment responses. The rate of decline of lymphocytosis is usually slower in patients with mutated IGHV and 13q14 deletion (less aggressive disease) and faster in patients with unmutated IGHV [10]. It does not require any specific management even when persistent for months [8].

\section{Drug Interactions}

Patients must be informed and monitored strictly for possible drug interactions for the possibility of increased toxicity or decreased efficacy [2].

\section{Special attention must be paid for}

i. Pharmacologic interactions between ibrutinib and P-glycoprotein substrates (digoxin and dabigatran), CYP3A4inhibitors and inducers including anti-arrythmic drugs (verapamil and amiodarone) and direct oral anticoagulants (apixaban, rivaroxaban), antimicrobials (azoles, macrolides, or rifampicin, [2] clarithromycin, ketoconazole or voriconazole) [3] and antiepileptic drugs (carbamazepine) [2].

ii. Options for patients requiring short period ( $\leq 7$ days) of moderate or strong CYP3A inhibitors, include i. withhold ibrutinib therapy and change to an alternative drug with a lesser degree of CYP3A inhibition, or ii. reduce the dose of ibrutinib to $140 \mathrm{mg}$ daily [11].

iii. Concomitant use of either AC or AP treatment with ibrutinib does not increase the risk of major bleeding [2]. Care should be exercised when initiating ibrutinib therapy in patients on anticoagulants and are at increased risk for experiencing major hemorrhagic events at baseline [7].

iv. Ibrutinib should be avoided in patients requiring dual or triple anti-coagulant and anti-platelet therapy [2].

v. Switch to low molecular weight heparin or direct oral anticoagulants if patient is receiving vitamin $\mathrm{K}$ antagonists. Concurrent use of warfarin is not recommended in cases with major hemorrhage [2].

a. Attention for co-incidental use of nonsteroidal antiinflammatory drugs and herbal supplements with CYP3A4 inhibitors or inducers [2].

b. Avoid grapefruit/juice, pomegranate/ juice, and Seville oranges with ibrutinib to prevent potentially significant interaction [11].

\section{When to stop Ibrutinib?}

Ibrutinib should be held for hematologic toxicity (grade 3 neutropenia with infection/fever or any grade 4) or non- 
hematologic toxicity (any grade 3) [11]. For grade $\geq 3$ infections, ibrutinib treatment should be paused and determine the cause of any unexplained fever or infection (e.g. CT scans). Ibrutinib should be reintroduced at the appropriate dose following infection resolution [3]. After improvement to grade 1 toxicity or baseline, the drug should be resumed at the starting dose. Of note, for each recurrence of toxicity, the daily dose should be reduced by $140 \mathrm{mg}$ (one capsule), and if toxicity persists after the doses have been reduced two times, therapy should be discontinued [11]. Ibrutinib must be stopped also 3-7 days, dictated by the risk level for bleeding, before [2] and postinvasive procedures, to allow time for the reversal of antiplatelet effects [3].

\section{Conclusion}

Ibrutinib has toxicity profiles that are different from those of immunochemotherapy. Bleeding and atrial fibrillation especially can pose complex treatment dilemmas. This is especially important in the light of continuous use of B-cell receptor inhibitors and should be taken into consideration when making individual treatment decisions.

\section{References}

1. Marini BL, Samanas L, Perissinotti AJ (2016) Expanding the armamentarium for chronic lymphocytic leukemia: A review of novel agents in the management of chronic lymphocytic leukemia. J Oncol Pharm Practice 23(7): 502-517.

2. Paydas S (2019) Management of adverse effects/toxicity of ibrutinib Crit Rev Oncol Hematol 136: 56-63.
3. Gribben JG, Bosch F, Cymbalista F, Geisler CH, Ghia P, et al. (2018) Optimizing outcomes for patients with chronic lymphocytic leukemia on ibrutinib therapy: European recommendations for clinical practice. Br J Haematol 180(5): 666-679.

4. Bosch F, Abrisqueta P (2015) Ibrutinib in CLL: 2 sides of the same coin. Blood 126(19): 2172-2173.

5. Herishanu Y, Katchman H, Polliack A (2017) Severe hepatitis B virus reactivation related to ibrutinib monotherapy. Ann Hematol 96: 689690.

6. de Jong J, Hellemans P, De Wilde S, Patricia D, Masterson T, et al. (2016) A drug-drug interaction study of ibrutinib with moderate and strong CYP3A inhibitors in patients with B-cell malignancy. Blood 128: 3964.

7. Jones JA, Hillmen P, Coutre S, Tam C, Furman RR, et al. (2017) Use of anticoagulants and antiplatelet in patients with chronic lymphocytic leukemia treated with single-agent ibrutinib. Br J Haematol 178: 286291.

8. de Weerdt I, Koopmans SM, Kater AP, van Gelder M (2017) Incidence and management of toxicity associated with ibrutinib and idelalisib: a practical approach. Haematologica 102(10): 1629-1639.

9. Wallace N, Wong E, Cooper D, Chao H (2016) A case of new-onset cardiomyopathy and ventricular tachycardia in a patient receiving ibrutinib for relapsed mantle cell lymphoma. Clinical Case Reports 4(12): 1120-1121.

10. Siddiqi T, Coutre S (2019) Chronic lymphocytic leukemia/small lymphocytic lymphoma. In: American Society of Hematology SelfAssessment Program. ( $7^{\text {th }}$ edn), pp. 700-718.

11. Lee C-S, Rattu MA, Kim SS (2016) A review of a novel, Bruton's tyrosine kinase inhibitor, ibrutinib. J Oncol Pharm Practice 22(1): 92-104.

\section{Your next submission with Juniper Publishers will reach you the below assets}

- Quality Editorial service

- Swift Peer Review

- Reprints availability

- E-prints Service

- Manuscript Podcast for convenient understanding

- Global attainment for your research

- Manuscript accessibility in different formats

( Pdf, E-pub, Full Text, Audio)

- Unceasing customer service

Track the below URL for one-step submission https://juniperpublishers.com/online-submission.php 